\title{
An Appraisal for the Need to Start Independent Research Institutes and Study Programs in Metabolic Engineering
}

\author{
Adhikarla Suryanarayana Rao \\ Department of Biotechnology, Bharathidasan University, Tiruchirappalli, India \\ Email address: \\ asraobio@gmail.com \\ To cite this article: \\ Adhikarla Suryanarayana Rao. An Appraisal for the Need to Start Independent Research Institutes and Study Programs in Metabolic \\ Engineering. Advances in Biochemistry. Vol. 9, No. 3, 2021, pp. 60-73. doi: 10.11648/j.ab.20210903.15
}

Received: August 6, 2021; Accepted: August 17, 2021; Published: August 24, 2021

\begin{abstract}
Metabolic engineering deals with manipulation of metabolic pathways for overproduction of desired metabolites $\&$ macromolecules and reduction/deletion of unwanted metabolites \& macromolecules. A large number of tools, techniques strategies \& methodologies are used in the metabolic engineering of microbial, plant \& mammalian/animal systems. Impressive advances have taken place in overproducing different categories of products Viz; biofuels, pharmaceuticals, neutraceuticals, cosmetics and many products relevant to different industries. In view of immense potential to bring about a next industrial biotechnology revolution, the need to establish specific goal oriented international research institutes on metabolic engineering has been emphasized apart from national institutes and study programs. Effort in this direction is environment friendly as well. At the outset readers are cautioned that manuscript is not a formal review or a discussion on the subject of "Metabolic Engineering" with respect to any specific topic or a living system but the purpose is to emphasize the need as mentioned above. Contents are adequate to think of this endeavor. Manuscript is a massive exercise after extensive scanning through the issues from the Journal "Metabolic Engineering") Elsevier, since its inception -1999 January (Volume 1) till January 2021 (Volume 63) (22 years). In all they constitute 63 volumes, 122 issues and 15,700 plus pages. Total number of articles (papers) in the above is approximately 1600 . Nearly $70 \%$ of these are with respect to "Microbial Systems". Next in importance comes the papers which have major focus on general tools, techniques, methodologies, strategies etc. Manuscript mentions and gives a glimpse of different approaches used to achieve the desired goals referring to a list of predominant host microbes, plants and mammalian/animal systems investigated. Readers are advised to initially go through the special issues of the journal.
\end{abstract}

Keywords: Industrial Biotechnology Revolution, Establishing Research Institutes, Microbes, Plants, Mammals, Metabolic Flux Analysis, Pharmaceuticals, Biofuels, Neutraceuticals

\section{Introduction}

\subsection{Note of Caution to Readers and Primary Purpose of the Manuscript}

This is not a formal review or a discussion on the subject of "Metabolic Engineering" with respect to any specific topic or a living system. As mentioned in the title, the purpose of this manuscript is to "emphasize the need to start independent research institutes and study programs across the world in the field of "Metabolic Engineering". To provide the required manpower, study programs may be started at Under Graduate and Post Graduate level of different time durations while the research institutes (private $\&$ public) at national and international level may be major goal(s) oriented. Further, the different contents of "Metabolic Engineering" can be incorporated in the course curriculum of already existing different life science programs and also in the related relevant disciplines/departments. Hence, academicians and science policy makers across the world should seriously ponder over to develop manpower and research in this field to provide the needed different valuable products to the society. In addition, advances in this field will be useful for further applications. Tools \& techniques etc used in this area of study will also be useful for studies other than metabolic engineering as well.

From the details presented here, both Corporates and Governments can assess the investment required, manpower 
from different disciplines required and the time dimension of the goals. The manuscript highlights the background which can justify and assist in the above endeavors. This paper has been written after extensive scanning through the issues from the Journal "Metabolic Engineering ") Elsevier, since its inception -1999 January (Volume 1) till January 2021 (Volume 63) (22 years). In all they constitute 63 volumes, 122 issues and 15,700 plus pages. Total number of articles (papers) in the above is approximately 1600 reflecting a greater average length of papers published. Bulk of this, nearly $70 \%$ pertain to Metabolic Engineering with respect to "Microbial Systems". Next in importance comes the papers which have major focus on general tools, techniques, methodologies, strategies etc They constitute roughly $18 \%$. (Of course there can be individual variations in categorizing the papers so. An important point to be noted here is, general tools, techniques, methodologies, strategies discussed in these articles are very much used in the bulk of the papers dealing with microbial, plant and animal/mammalian systems). Metabolic engineering with respect to plant and animal/mammalian systems constitute a smaller fraction. An important point to be noted by the readers is papers related to metabolic engineering were being published / are being published in various other journals too, other than the journal Metabolic Engineering. However, this journal focuses exclusively on metabolic engineering with different living systems and the papers published do quote other papers concerning metabolic engineering published in various other journals. Papers in this journal are adequate enough to serve the endeavors mentioned above.

\subsection{Nature and Scope Metabolic Engineering}

Readers should be clear as to what is "Metabolic Engineering"? A meaningful way to define "Metabolic Engineering" is to know in the words of the journal itself (Metabolic engineering, Elsevier) as mentioned in the aim and scope of the journal. It is concerned with the research papers "on the direct modulation of metabolic pathways for metabolite overproduction or the improvement of cellular properties. Papers describe native pathway engineering" [1]. To make it more explicit two more definitions in different words: Metabolic engineering is defined as "the improvement of cellular activities by manipulation of enzymatic, transport, and regulatory functions of the cell with the use of recombinant DNA technology" [2] and "Metabolic engineering is the practice of optimizing genetic and regulatory processes within cells to increase the cell's production of a certain substance. These processes are chemical networks that use a series of biochemical reactions and enzymes that allow cells to convert raw materials into molecules necessary for the cell's survival. Metabolic engineering specifically seeks to mathematically model these networks, calculate a yield of useful products, and pin point parts of the network that constrain the production of these products. Genetic engineering techniques can then be used to modify the network in order to relieve these constraints. Once again this modified network can be modeled to calculate the new product yield". The ultimate goal of metabolic engineering is to be able to use these organisms to produce valuable substances on an industrial scale in a costeffective manner." [3]. To add - "Metabolic engineering" is concerned with the manipulation of metabolic fluxes to accordingly divert and enhance the formation of desired products or elimination of undesired products. In the living systems particularly microbes both native and non-native products are produced both by manipulating native genes and/or manipulating and introducing heterologous genes form different other living systems towards the desired product formation in desired levels.

Metabolic Engineering is an endeavor involving many Tools/Techniques/Strategies Mechanisms/Methodologies Employed in Metabolic Engineering (Section 2 for a comprehensive list). Here, for an initial background and appraisal a few strategies are mentioned in an alphabetical order and does not in anyway reflect their relative importance or extent of applicability. They are Viz; activation of dormant biosynthetic pathways; adaptive laboratory evolution; altering the compartmentalization of metabolites; Balancing Cellular Redox Metabolism- altering the levels of reducing power -NAD/NADH, NADH/NADPH and NAD/NADP ratios; altering the ratios of Co-A/acetyl Co-A; Construction and Use of Different Vectors for transformation of Different Microorganisms, Plants, and Organelles; Different Types of Microbial fermentations; Disruption of unwanted pathways; Eliminating toxic intermediates/products in the cell; Enhancing production of rate limiting enzymes; Genetic Transformation of plants (Transgenic Plants) by Agrobacterium rhizogenes / Agrobacterium tumefaciens / Particle Bombardment/ Electroporation / Viral mediation; Manipulating Membrane Transporters; Manipulating Pathways in different organelles/compartments of the cell; Manipulating transcription regulators; Manipulation of plasmid copy numbers; Mathematical modeling; Optimizing codon usage; Optimizing promoter strength; over expression of native and /or heterologous enzymes/proteins; Precursor balancing; Process optimization; Recombinant DNA technology/Gene cloning and Expression Studies/ Over expression of native and Heterologous genes; Reducing apoptosis of the cells; Tissue Culture Techniques / Cell culture/tissue Culture/Organ culture / Intact Plants; Use of different promoters -Constitutive/ Inducible/Strong/ Heterologous / Tissue specific/Hybrid; Use of different Transcription Factors; Use of Elicitors for Over production of Desired Metabolites; Use of labeled Precursors; Use of Mutant Strains; Use of Recombinant Plasmids.

\section{Tools/Techniques/Strategies Mechanisms/Methodologies Employed in Metabolic Engineering}

A special issue [4] highlights as follows: Metabolic Flux Analysis, Machine Learning-based Modeling, Combinatorial metabolic pathway assembly approaches, High-Throughput 
Screening and CRISPR-derived genome editing technologies. However, many more are covered in other issues and explained more specifically which need to be known to accomplish the goals as outlined. They may be listed as follows:

Assessing data Structure and Class Homogeneity by Cluster Analysis; Antisense Inactivation; BICES (Biomassinducible chromosome based expression system); Boolean function; Cofactor Manipulation; Cell free metabolic engineering; Combinatorial genetic manipulation/metabolic engineering; Comparative genomics; Computational Analysis; Confocal / Transmission / Electron Microscopy; CRISPRi / CRISPRa ( $\mathrm{i}=$ interference; $\mathrm{a}=$ activation); CRISPR/Cas 9 -facilitated multiplex pathway optimization technique (CFPO technique) / CRISPR/Cas 9 Genome Editing system; Cybernetic approaches; (Continued below).

DNA Microarray Detection; Dynamic Flux Balance analysis; Dynamic Optimal Metabolic Control; Engineering the bacterial shapes; Evolutionary metabolic Engineering; Ensembling Modeling Framework; Extensive Modeling for Robust Analysis (EBRA); Flow Cytometric Analysis; Functional genomics /High-Throughput techniques for measuring Transcriptome, Proteome and Metabolome; Gene Knock Outs; Gene Silencing; Gene Therapy; Genome scale modeling; Genome wide analysis; Immuno blot Analysis; Introduction of additional copies of Transcription factors; Use of Stable/labeled isotopes $-{ }^{13} \mathrm{C},{ }^{14} \mathrm{C},{ }^{1} \mathrm{H},{ }^{2} \mathrm{H}_{2} \mathrm{O}, \mathrm{H}_{2}{ }^{18} \mathrm{O}$, ${ }^{31} \mathrm{P}$; Isotopomer Spectral Analysis (ISA); Improving cell viability and cell growth; Iterative / Precision / Systems / Combinatorial Metabolic Engineering; LC-ESI-MS/MS; Linlog kinetics approach; MALDI-MS/ MALDITOF/MALDI-TOF/TOF; Knock out of unwanted genes; (Continued below).

Manipulating Cofactors; Mean Hypothesis Testing; Membrane Engineering Strategy; Metabolic Control Analysis (MCA) / Inverse Metabolic Control Analysis (IMCA)/NonLinear Metabolic Analysis; Metabolic Flux Analysis (MFA) /

13 CMFA / FBA (Flux Balance Analysis); Metabolic Modeling; Metabolic Transistor Strategy; Mixed Integer Linear Program (MILP); Multiplex Automated Genome Engineering (MAGE); Multiplex Navigation of Global regulatory networks (MTNR); Multiplex Site specific Genome Engineering (MSGE); Non-Equilibrium Thermodynamics; Parallel Capillary Electrophoresis; Predicting metabolic fluxes in native and engineered organisms by mathematical modeling; Proteomics/targeted proteomics/ Principal component analysis of proteomics (PCAP); qPCR (quantitative PCR) and RT-qPCR/ qRT-PCR (Quantitative Real Time-PCR)/ PCR analysis; Promoter Replacement; (Continued below).

Quantitative Trait analysis; RNA sequencing; Signal Transduction Dynamics; Study of Bioenergetics; Study of Membrane Transporters / Overcoming Membrane barriers; Stochastic Simulation; Study of Compartmentalization of Enzymes and metabolites; Study of Quantitative Trait Loci in Plant Systems; Synthetic Biology; Targeted Genetic Modification /Registered Mutagenesis; Targetron
Technology; Tendency Modeling Approach; Thermodynamics and Kinetic Analysis of Metabolic pathways; Transcriptional Reprogramming; Transcriptional Profiling; Transient isotopic Flux Analysis and Kinetic Modeling; Use of GC-MS/ GC-C-IRMS (Gas Chromatography-combustion-isotope ratio mass spectrometry) / LC-MS/ HPLC and 1 D, 2 D NMR; Use of specific Biosensors; Use of Transcriptional regulators /factors; Using Different Algorithms; Whole Genome Sequencing/ Genome Wide Analysis.

In addition to the above mentioned (Section $1.2 \& 2$ ) routine techniques like electrophoresis/different blots, different bioreactors \& fermentations, spectrophotometry etc are used besides assays of different enzymes, estimations of different metabolites and other techniques associated with the above which are mentioned in the papers published.

\section{Metabolic Engineering of Microbes}

As mentioned above, bulk of the papers -nearly $70 \%$ pertain to microbial systems. Since long, producing chemicals of therapeutic and industrial relevance in large amounts economically from microbes has been considered as an attractive alternative strategy. Both native and non-native products are produced in different microorganisms and different microbial products can also be produced in plants by metabolic engineering. A recent special issue [5] exclusively concerned with microbial hosts mentions eleven different predominant microbial hosts Viz; Escherichia coli, Sacchromyces cerevisiae, Corynebacterium glutamicum, Cyanobacterial sps, Yarrowia lipolytica, Streptomyces sps, Bacillus subtilis, Clostridium sps, Pseudomonas putida, Pichia pastoris and Zymomonas mobilis. Besides above many others are studied in the numerous papers published (mentioned in the text).

\subsection{Products and Processes of Microbial Metabolic Engineering}

Studies on microbial metabolic engineering broadly covers: Manipulation of central/primary metabolism Viz; Glycolysis, TCA cycle, Pentose phosphate pathway, BetaOxidation Pathway, Fatty acid biosynthesis, Manipulation of: central nitrogen metabolism---amino acid biosynthesis and other nitrogenous compounds; secondary metabolism (producing plant specific secondary metabolites in different microbial hosts); fatty acid chain length, Manipulating MEP (methyl erythritol phosphate) and MVA (Mevalonic acid) pathway to alter isoprenoid levels; Co-factor levels (NADH/NAD, NADPH/NADP \& NAD/ NADP ratios and Co-A/Acetyl Co-A ratios). Increasing the levels of precursors for building blocks of industrially relevant products, Expression of recombinant proteins, plant hormones, sugar alcohols, biofuels, polyketides, aromatic compounds, oleochemicals and pharmaceutically active compounds [6-14].

A detailed list of the products of microbial metabolic 
engineering is as follows (Note of caution - List is confined to the journal issues browsed as mentioned above and no claim is made that there are no omissions at all. Products are listed here in alphabetical order and in an order that does not in anyway reflect their importance or extent of their investigation:).

Of the total papers on microbes nearly $40 \%$ are on Escherichia. coli followed by Sacchromyces cerevisiae and Corynebacterium glutamicum but these two fall much short in number of papers compared to E. coli. and others are still less. Readers should be aware that the same product may be produced in different microbes using similar or different approaches and even in the same microbe by different ways. In a nut shell the products and their process of production are as follows.

\subsection{E. coli}

Increasing the levels/production of the following: recombinant protein production by decreasing acetate accumulation by cloning Bacillus acetolactate synthase; 1,2 propanediol, isoamyl acetate and succinate by manipulating the cofactor ratios NADH/NAD and acetyl Co-A/Co-A by cloning the enzymes nicotinic acid phosphoribosyl transferase (NAPRTase), encoded by the pncB gene, alcohol transferase and pyruvate formate lyase; NADH availability by over expressing NAD dependent formate dehydrogenase from Candida boidinii (Alteration in NADH/NAD ratios alters metabolic fluxes and production of metabolites as NAD is involved in nearly 300 oxidation-reduction reactions in the cell); NADPH availability by substituting native $E$. coli NAD-dependent Glyceraldehyde-3-phosphate dehydrogenase by NADP-dependent Glyceraldehyde-3phosphate dehydrogenase from Clostridium acetobutylicum which enhances NADP dependent metabolic pathways and their products; tetrahydrobiopterin (BH4) -an important drug to treat atypical hyerphenylalaninemia - by a combination of different strategies Viz; cloning and over expressing the genes for the enzymes, GTP cyclohydrolase 1 (GCH1), 6pyruvyltetrahydropterin synthase (PTPS), sepiapterin reductase (SPR), de novo GTP supply by the addition of another gauBA operon and further increasing the activity of GCH1 by cloning mtrA gene (DNA binding response regulator); Shikimic acid (an important starting material for the production of anti-viral drug Tamiflu) by blocking Shikimate kinase and to dephosphorylate already formed Shikimate 3-phisphate by phosphatases; succinate production in a penta mutant (knocking out of five genes) which creates an active glyoxylate cycle followed by over expression of phosphoenol pyruvate carboxylase and inactivation of phosphoenol pyruvate sugar phosphotransferase system; (Continued below).

Lycopene production in triple gene knockout mutants thereby lacking NADP specific glutamate dehydrogenase, pyruvate dehydrogenase and transaldolase $\mathrm{B} /$ fumarate dehydrogenase and overexpression of 1-deoxy-d-xylulose-5phosphate synthase, isopentenyl diphosphate delta isomerase and isp genes involved in metabolizing 1-deoxy-d-xylulose-5- phosphate into Isopentenyl pyrophosphate; Glucosamine and $\mathrm{N}$-acetyl glucosamine by over expression of glucosamine synthase and $\mathrm{N}$-acetylglucosamine transferase and decreasing catabolic genes of glucosamine; beta-carotene production by replacing native promoter of the isoprenoid genes with the strong bacteriophage T5 promoter; d-phenylglycine -an important precursor for semisynthetic penicillins and cephalosporins - by over expressing heterologous enzymes, hydroxymandelate synthase, hydroxymandelate oxidase and hydroxyphenylglycine aminotransferase; Increasing parahydroxycinnamic acid by over expressing Tyrosine ammonia lyase from yeast Rhodotorulas glutinis; p-hydroxystyrene by over expressing the enzymes p-Hydroxycinnamic acid decarboxylase from Bacillus subtilis and Lactobacillus plantarum and bifunctional Phenylalanine ammonia lyase /Tyrosine ammonia lyase from the yeast Rhodotorula glutinis; Simvastatin (cholesterol lowering drug) by using BioH mutant strain which cannot hydrolyse dimethylbutyryl-S-methylmercaptopropionate (DMB-S-MMP) which is required for high levels Simvastatin biosynthesis; (Continued below).

1-Propanol was achieved by overexpressing heterologous 2-ketoacid decarboxylase (Kivd) from Lactococcus lactis and alcohol dehydrogenase 2 (ADH2) from Saccharomyces cerevisiae. Further butanol production also was achieved simultaneously by over expressing native threonine dehydratase and leucine biosynthetic enzymes and elimination of competing pathways by deletion of homoserine O-succinyl transferase, threonine dehydrogenase and catalytic subunits acetohydroxy synthase I \& III. Both production of 1-propanol and 1-Butanol could be achieved by these strategies; free fatty acids by knocking out the endogenous fadD gene, which encodes an acyl-CoA synthetase, to block fatty acid degradation, heterologous expression of a plant thioesterase to increase the abundance of shorter chain fatty acids, over-expressing ACC (acetyl CoA carboxylase to increase malonyl Co-A and releasing feedback inhibition caused by long-chain fatty acyl-ACPs through over expression of an endogenous thioesterase. All these strategies increase the production of free fatty acids which are in turn useful for biodiesel (methyl esters of fatty acids) production; odd chain fatty alcohols by expressing alpha-dioxygenase fatty acyl carrier protein (-ACP) thioesterase and aldehyde reductase or aldehyde decarbonylase; Ethanol from glycerol by over expressing glycerol dehydrogenase and dihydroxyacetone kinase and inactivating fumarate reductase and phosphoacetyl transferase to minimize the by-products succinate and acetate respectively. A strain was also constructed which forms fumarate along with ethanol by inactivating formate lyase; (Continued below).

Amorpha-4,11-diene -a precursor of anti-malarial drug artemisinin - by over expression of rate limiting enzymes mevalonate kinase and amorphadiene synthase; production of plant specific phenylpropanoids Viz; 4-coumaroylserotinin, 4-Coumaroyloctopamine and 4-coumaroyltyramine by over expressing 4-coumarate: ligase from Arabidopsis thaliana and serotonin N-hydroxy-cinnamoyl transferase from pepper 
plants (Capsicum annuum); mannitol production by over expressing heterologous mannitol dehydrogenase, formate dehydrogenase and a sugar facilitator protein (permease) from Leuconostoc pseudomesenteroides; enhancing 2pyrone-4,6-dicarboxylic acid (an important building block for the synthesis of many polymers) by cloning and expression of heterologous genes from Emericella niger, Pseudomonas putida and Sphingobioium sp SYK-6; Malonyl Co-A (a major building block for natural products with therapeutic significance) by over expression of acetyl Co-A carboxylase and acetyl-CoA synthase, inactivation of malonyl-CoA-consuming fatty acid synthesis and eliminating competing pathways leading forming of acetate and ethanol by knocking out phosphotransacetylase and acetate kinase and bifunctional alcohol/aldehyde dehydrogenase; (Continued below).

Naringenein (a flavanone precursor for other flavonoids) from glucose by expressing heterologous plant and microbial enzymes, tyrosine ammonia lyase, 4-coumarate Co-A ligase, Chalcone synthase and chalcone isomerase; 5-amino levulinic acid by expressing glutamyl-tRNA reductase from Salmonella arizona and glutamate -1-semialdehyde aminotransferase and threonine /homoserine exporter from $E$. coli itself; copolymer of 3-hydroxypropionate and 4hydroxybutyrate (P(3HP-co-4HB)) by expressing five heterologous genes encoding 4-hydroxybutyrate coenzyme A transferase, ACS domain of propionyl-Co-A ligase, dehydratase, aldehyde dehydrogenase, and polyhydroxyalkanoate synthase; free fatty acids by expressing acyl-ACP thioesterase from Ricinus communis; increasing 1-propanol production by expressing feedback resistant threonine dehydratase, citramalate synthase, acetate kinase A / propionate kinase II, alcohol dehydrogenase and deleting stationary phase sigma factor and competing metabolic pathways for threonine; Polyhydroxybutyrate and hydrogen production by expressing hydrogenase 3 and /or acetyl- CoA synthetase; hydroxylated tyrosine by introducing an alternative cofactor tetrahydromonapterin from $E$. coli itself in place of tetrahydrobiopterin; (Continued below).

Acetone by a novel pathway in specific strains of E. coli by expressing heterologous thiolase, acetoacetate decarboxylase, and thioesterase; fatty alcohols by over expressing native alcohol dehydrogenase and heterologous fatty acyl-CoA/ACP reductase; 2,3-Butanediol by incorporating several heterologous genes and process optimization; 3-hydoxy propionic acid by over expression of glycerol-3-phosphate kinase, glycerol transport facilitator protein, deletion of glycerol pathway repressor protein and reducing the by-products acetate and 1,3-propane diol; Fatty aldehydes are converted to alkanes by the enzyme aldehyde deformylating oxygenase. However, aldehyde reductases reduce this conversion. Hence, increased production of alkanes was achieved by deleting the genes for aldehyde reductases; Triglyceride by expressing five genes in fatty acid formation/utilization along with wax ester synthase/ acyl-Coenzyme A: diacylglycerol acyltransferase; myoinositol production from Glucose 6-phopshate by controlled degradation of the native enzyme phosphofructo kinase; polyhydroxybutyrate by substituting glucose with gluconate as a substrate under Nitrogen limiting conditions; Nacetylglucosamine by expressing heterologous enzymes Nacetylglucosamine 2-epimerase and Glusosamine -6phosphate acytltransferase; fatty acid production by expression of a transcription factor FadR which regulates the transcription of many genes involved in fatty acid synthesis, degradation and membrane transport; medium fatty acid chain length (C12-C14) polyhydroxyalkaonate primarily of 3-hydroxydodecanoate monomers. This was achieved by over expressing acyl-ACP thioesterase, enoyl-CoA hydratase and medium chain length -PHA polymerase in a mutant $E$. coli strain; (Continued below).

Xylitol from Xylose by combining different strategies Viz; over expressing Xylose reductase, eliminating catabolite repression by disrupting phosphoenolpyruvate dependent glucose phosphotrasferase system and deleting Xylose catabolic/utilizing enzymes to other products; Aromatic compounds (chorismate derivatives) in a phenylalanine over producing strain in which reactions from Phosphoenolpyruvate to Pyruvate were inactivated; isopentenol by expressing phosphomevalonate decarboxylase and phosphatase; two carbon compounds ethylene glycol and glycolate form pentose Xylose by manipulating the enzymes Lactaldehyde dehydrogenase and Lactaldheyde reductase; branched chain fatty acids by restoring -2-oxyacid dehydrogenase lipolyation; long chain fatty alcohols primarily by deleting NADP dependent aldehyde reductase, optimized expression of acyl-ACP reductase and further by process optimization; odd chain fatty alcohols by expressing alpha-dioxygenase fatty acyl carrier protein (-ACP) thioesterase and aldehyde reductase or aldehyde decarbonylase; monolignols, p-coumaryl, caffeyl and coniferyl alcohols, (precursors for Lignin biosynthesis) by introducing hydroxylase with better properties and methyltransferase and adopting a consortium (Co-culture); serine by adaptive laboratory evolution; 3,4 dihydroxybutyric acid by from Xylose by introducing a novel five step biosynthetic pathway which can also produce another useful chemical 3, Hydroxy-gamma-butyrolactone; lactams Viz; four carbon (butyrolactam), five carbon (valerolactam) and six carbon (caprolactam) using omega -amino acids and heterologous beta-alanine Co-A transferase; isopentenol by using an unique mevalonate diphosphate decarboxylase under aeration limited conditions; (Continued below).

3-polyhydroxybutyrate by weakening the rigidity of the cell by manipulating cell wall synthesis by selectively down regulating genes in cell wall biosynthesis; assimilation of methanol by co-utilization of threonine and deletion of leucine-responsive regulatory protein; pyrogallol by overexpression of 2,3-DHBA (dihydroxybenzoic acid) synthase and 2,3-DHBA 1-monoxygenase; glycolate by overexpressing glycolate pathway enzymes isocitrate lyase, isocitrate dehydrogenase kinase/phosphatase and glyoxylate reductase in specific strains of $E$. coli; malate from Xylose by combining different strategies Viz; overexpressing D- 
tagatose 3-epimerase, L-fuculokinase, L-fuculose-phosphate aldolase, and aldehyde dehydrogenase A, Knocking out of genes encoding malic enzyme, malate dehydrogenase, and fumarate hydratase to eliminate malate consumption and over expression of glycolate oxidase and malate synthase to intensify the conversion of glycolate to malate,; 3Hydroxypropionic acid by introducing its biosynthesis module into a Fatty acid -utilizing strain, and further optimizing the process; plant flavonoids baicalein and scutellarein by combining different approaches Viz; engineering plant flavonoid biosynthetic pathway genes from five different species Viz; phenylalanine ammonia lyase from Rhodotorula toruloides, 4-coumarate-coenzyme A ligase from Petroselinum crispum, chalcone synthase from Petunia hybrida, chalcone isomerase from Medicago sativa and an oxidoreductase flavone synthase I from $P$. crispum, incorporating a 22-aa N-terminal truncated flavone C-6 hydroxylase from $S$. baicalensis and partner P450 reductase from Arabidopsis thaliana and by optimization of malonylCoA availability; (Continued below).

Aspartate and beta alanine by over expressing L-aspartate aminotransferase for producing L-aspartate and L-aspartatea-decarboxylase for producing $\beta$-alanine; styrene by combining different strategies Viz; selecting a strain producing high styrene, designing and constructing a transcription regulator library targeting 54 genes with 85,420 mutations, and selecting the best mutant for high styrene production; 3-hydroxyvalerate from glucose and glycerol by combining different strategies Viz;. Activation of the four gene sleeping beauty mutase (Sbm) operon sbm-ygfD-ygfGygfH to enable the intracellular formation of non-native propionyl-CoA, manipulating biosynthetic enzymes for the utilization of glycerol into $3-\mathrm{HV}$, and redirecting more dissimilated carbon flux from the tricarboxylic acid (TCA) cycle to the Sbm pathway, resulting in an enlarged intracellular pool of propionyl-CoA. and therefore subsequently $3-\mathrm{HV}$ biosynthesis and deregulating glyoxylate shunt and blocking the oxidative TCA cycle; acetate accumulation by inactivating citrate synthase activity; Valine by a combination of different approaches Viz; heterologous introduction of a feedback-resistant acetolactate acid synthase along with two more enzymes in the valine synthetic pathway, introduction of an heterologous exporter for efficient efflux of valine, knockout of GTP pyrophosphokinase, introduction of a ppGpp 3'pyrophosphohydrolase mutant to facilitate the glucose uptake process and improving the redox cofactor balance by replacing acetohydroxy acid isomeroreductase by a NADHpreferring mutant, and branched-chain amino acid aminotransferase by leucine dehydrogenase from heterologous sources; succinate by deleting alcohol dehydrogenase, acetate kinase-phosphotransacetylase, lactate dehydrogenase, formate acetyl transferase and phosphoenolpyruvate dependent sugar phosphotransferase and over expression of Phosphoenolpyruvate carboxykinase; succinic acid production from different carbon sources Viz; fructose, sucrose, mixture of glucose and fructose, mixture of fructose, glucose and sucrose and sucrose hydrolysis solution by using a mutant strain containing heterologous pyruvate carboxylase and sucrose uptake and catalyzation genes; Tyrosine by over expressing shikimate dehydrogenase and shikimate kinase; (Continued below).

In addition to the above the following processes and products were investigated as much as above mentioned in $E$. coli. To minimize length and for simplicity, in the above all products have been covered using the expression in the beginning "Increasing the levels/production of the following "To reflect other different expressions in the papers published, aspects have been mentioned below accordingly Manipulating, Over expression, Engineering etc Different aspects studies are as follows: flexibility and limits of $E$. coli metabolism was investigated by deletion of 20 core enzymes in central metabolism; Manipulating the following: ethanol and lactate levels by over expressing heterologous glycolytic enzymes, phosphofructo kinase (Lactobacillus bulagricus) and Pyruvate kinase (Bacillus stearothermophilus); levels of lactate and carbon flux in primary metabolism by over expressing and deleting lactate dehydrogenase; levels and metabolic directional flow of pyruvate as influenced by the activities of Pyruvate formate lyase and Lactate dehydrogenease; glucose and acetate utilization rates through TCA cycle, glyoxylate shunt and PPP, by knocking out the gene zwf which codes for Glucose -6-phosphate dehydrogenase; alkane production by deletion of endogenous aldehyde reductase and over expression of fatty alcohol oxidase. Further, increase in alkane production was achieved by manipulating the process of fatty acid biosynthesis, lipid degradation and electron transfer system; Stress responses studied due to over expression of recombinant proteins as reflected in the formation of SOS, stationary phase and heat shock genes and reduced growth rate, inclusion body formation, plasmid instability and lysis;

Engineering the following pathways / metabolite production: Hyaluronic acid biosynthesis by over expressing heterologous Hyaluronic acid synthase from Streptococcus pyrogenes. In addition to glucose, sorbitol also can be used for the production of hyaluronic acid; reduction in acetate accumulation by inactivating phosphoenolpyruvate: sugar phosphotransferase system (PEP-PTS) to facilitate increased recombinant protein production; Degradation of cyclohexane carboxylate $(\mathrm{CHC})$ to form pimeloyl $\mathrm{Co}-\mathrm{A}$ a precursor for Biotin synthesis has been facilitated by cloning genes for degradation of $\mathrm{CHC}$ from Rhodopseudomonas palustris after reducing their $\mathrm{GC}$ content. This strategy has increased Biotin production; 1-Butanol biosynthesis and increasing 1-Butnaol by cloning and over expressing genes from Clostridium acetobutylicum coding for thiolase, 3-hydroxybutyryl Co-A dehydrogenase, crotonase, electrontransfer flavoprotein, aldehyde/alcohol dehydrogenase and acetyl Co-A acetyl transferase and deletion of native host (E. coli) enzymes, lactate dehydrogenase and fumarate reductase; cis, cisMuconic acid pathway by expressing isochorismate synthase and isochorismate pyruvate lyase (which contribute to phenylalanine production) into a Salicylic acid 
overproducing strain and also expressing salicylate -1monooxygenase and catecho 1,2 -dioxygenase to convert salicylic acid into muconic acid; Entner-Doudoroff (ED) Pathway to increase NADPH supply and ultimately terpenoid production; Beta-carotene production was engineered by improving ATP synthesis, Pentose phosphate pathway and TCA cycle; enhanced production of 3-propionic acid by over expressing heterologous 1-aspartate-alpha-decarboxylase, replacing native promoters of aspartate ammonia lyase \& acetyl Co-A synthetase genes with strong trc (hybrid promoter); (Continued below).

Acrylic acid biosynthetic pathway from glucose via 3hydroxypropionate by introducing seven different novel enzymes; novel pathway from Pyruvate to 2,3 Butane diol by expressing three heterologous enzymes and by adding glucose and catalytic amounts of cofactors NAD and ATP to the extracts of this strain high levels of 2,3 Butane diol can be synthesized; dipicolinic acid production by over expression of specific aspartate kinase, dipicolinate synthase and phosphoenolpyruvate carboxylase; metabolic engineering for the production of 5-Hydroxytryptophan by expressing human tryptophan hydroxylase I and supplying cofactor tetrahydrobiopterin (BH4) by reconstituting human BH4 biosynthesis and regeneration; astaxanthin (xanthophylls) metabolically to a high concentration by expressing five heterologous genes heterologous from Pantoea ananatis and truncated BKT gene from Chlamydomonas reinhardtii; uridine formation by integrating eight genes in the pyrimidine operon of Bacillus subtilis into the yghX locus of E. coli W3110 Further production has been increased by knocking, five genes involved in uridine catabolism and by manipulating genes for improving the uridine precursors carbamyl phosphate, aspartate, and 5'phosphoribosyl pyrophosphate; (Continued below).

Overexpression of the following: rspAB operon (involved in homoserine degradation) suggested as a strategy to increases recombinant protein production; pantothenate kinase increases ratio of Co-A/acetyl Co-A which resulted in increased production of isoamyl acetate which was facilitated by the over expression of alcohol acetyl transferase. By altering the levels of Co-A/acetyl Co-A more than 100 metabolic reactions can be influenced; alteration of glucose and acetate utilization rates through TCA cycle, glyoxylate shunt and PPP, by knocking out the gene zwf which codes for Glucose -6-phosphate dehydrogenase; transhydrogenase and NAD kinase on chromosome increases NADP supply and Isobutanol production;

By mathematical modeling phosphoenol pyruvate carboxylase, Aspartate kinase and Glutamate dehydrogenase were suggested as targets for increasing Lysine production; production of copolymer Poly(3-hydroxybutyrate-co-4hydroxybutyrate) from glucose by expressing following enzymes from Ralstonia eutropha, beta- ketothiolase; NADPH dependent acetoacetyl-CoA reductase, PHA synthase, succinate semialdehyde dehydrogenase, 4-hydroxybutyrate dehydrogenase, CoA transferase; and succinate semialdehyde dehydrogenase of E. coli; systematic optimization of IgG antibody expression by suitably influencing transcription, translation and in vivo protein folding; Monoterpene Limonene and its hydroxylated compound Perillyl alcohol (anti-cancer agent) have been produced by heterologous expression of Mevalonate pathway and Limonene synthase coupled with Cytochrome P-450: Antibiotic Erythromycin analogue was produced by increasing the production of propionyl Co-A and (2S)- methylmalonyl Co-A by expressing propionyl Co-A synthetase and propionyl Co-A Carboxylase and also by a separate pathway by expressing malonyl-CoA ligase to increase medium chain (C12-C14) fatty acids; (Continued below).

Many genes from plants and bacteria were used to make four phenylpropanoids, three stilbenoids and three curcuminoids; achieved synthesis of co-polymer of 3hydroxybutyrate and 3-hydroxypropionate (PHB3HP) by introducing several heterologous genes from different bacteria; Ectoine (1,4,5,6-tetrahydro-2-methyl-4-pyrimidinecarboxylic acid), pathway constructed and its production increased by different strategies Viz; introducing heterologous L-2,4diaminobutyric acid transaminase, L-2,4-diaminobutyric acid $\mathrm{N} \gamma$-acetyltransferase, L-ectoine synthase, deletion of bifunctional aspartokinase/homoserine dehydrogenase, feedback resistant aspartokinase and replacement of native promoter of phosphoenol pyruvate carboxylase by hybrid trc promoter; Synergy between MEP and MVA pathways -that is simultaneous over expression of both the pathways - has been found to increase isoprenoid production. In specific lycopene production has been demonstrated; construction of 4-HB overproducing $E$. coli strains optimized for microaerobic cultivation by amplifying both oxidative and reductive TCA branches and glyoxylate shunt; production of naringenin from methanol by introducing heterologous NAD dependent Methanol dehydrogenase and Ribulose monophosphate enzymes; Achieving antibody production in a strain over expressing a mutated global sigma factor RpoD along with chaperone co-expression; flexibility and limits of $E$. coli metabolism was investigated by deletion of 20 core enzymes in central metabolism; Benzoic acid production achieved by engineering a plant beta-oxidation pathway and a synthetic pathway and was further optimized by enhancement of the precursor availability, removal of competitive reactions, transporter engineering, and reduction of byproduct formation.

\subsection{Sacchromyces Cerevesiae}

Increasing the levels/production of the following: ethanol by increasing ATP \& NADH consumption by over expressing glutamine synthetase and deleting NADPH consuming glutamate dehydrogenase; increasing ethanol and reduction of Xylitol, glycerol and acetate by expressing a fusion protein of Xylose reductase and Xylitol dehydrogenase from an heterologous source; Ethanol production from Xylose by introducing heterologous Xylose reductase and Xylose dehydrogenase and over expressing endogenous Xylulokinase under both aerobic and anaerobic conditions; ethanol from cellulose by expressing heterologous endoglucanse and beta-glucosidase; para- 
hydroxycinnamic acid by over expressing the enzymes Cinnamic -4-hydroxylase (a cytochrome p-450 monooxygenase) and Cytochrome P-450 reductase from Helianthus tuberosus; glycerol by over expression of Glycerol phosphate dehydrogenase in triose phosphate isomerase defective mutant and NAD -aldehyde dehydrogenase and deletion of NAD -alcohol dehydrogenase; aromatic amino acid biosynthesis (Phenyl alanine and Tyrosine) by expressing tyrosine feedback insensitive 3-deoxy-d-arabino-heptulosonate-7-phopshate synthase which was further increased when combined with feedback insensitive chorismate mutase; producing ethylene by cloning and expression of Ethylene forming enzyme from Pseudomonas synringae which forms ethylene using alphaketoglutarate and arginine; Dihydroxyacetone by expressing heterologous NAD dependent glyceraldehydes dehydrogenase and deletion of Dihydroxyacetone Kinase; NADPH supply by over expressing malic enzyme resulted in decreased pentose phosphate pathway and alteration in the levels of redox factors. In one strain because of lack of mitochondrial target sequence malic enzyme was apparently accumulated in the cytosol itself; glycerol by deletion of pyruvate decarboxylase, NADH dehydrogenase and respiratory chain linked glycerol-3-phosphate dehydrogenase. Additional increase could be achieved by supplementing formate to glucose; succinic acid by deleting the enzyme succinate dehydrogenase; reseveratrol by modifying bacterial Tyrosine ammonia lyase gene codons to be preferred by yeast and expressing arabinose transporter; fatty acid derived metabolites by over expressing acetyl CoA carboxylase, fatty acid synthases $1 \& 2$; Triacetyl lactone by over expressing Gerbera hybrida 2-pyrone synthase and influencing several genes in primary metabolism and increasing precursor and co-factor supplies; (Continued below).

Dihydrochalcones by over production of enoyl-Co-A reductase and elimination of the major by-product naringenin by expressing chalcone synthase chalcone synthase and expressing enzymes relevant for production of phloretin the first committed dihydrochalcone; isoprenoids by expressing and increasing the activity of plant isoprene synthase; monoterpene Geraniol by expressing geraniol synthase, farnesyl diphosphate synthase, truncated 3-hydroxy-3methylglutaryl Co-A reductase and isopentenyl diphosphate isomerise; valine biosynthesis by mutating the regulatory subunit of acetohydroxy acid synthase (AHAS) and making it less sensitive to feedback inhibition by valine; SCA (simvastatin acid) by introducing six heterologous biosynthetic genes and the acyl-donor dimethylbutyryl-Smethyl mercaptopropionate (DMBSMMP). Further increase in levels were obtained by isolating a variant which has a very high activity of acyltransferase towards DMB-SMMP in the synthesis of SVA from MJA; Lycopene by over expressing key genes associated with fatty acid synthesis and triacylglycerol (TAG) production, followed by modulation of TAG fatty acyl composition by overexpressing a fatty acid desaturase and deletion of Seipin, which regulates lipid- droplet size; MIAs (Monoterpene Indole alkaloids) by increasing their precursor strictosidine levels. A variant strain with enhanced production of the monoterpene precursor geraniol was engineered and further the geraniol biosynthetic pathway was targeted to the mitochondria to protect the GPP pool from consumption in the cytosol. Its conversion to strictosidine was achieved by expressing Catharanthus gernaiol-8-hydroxylase, geraniol oxidoreductase and iridoid synthase which synthesize intermediates in the biosynthesis of Strictosidine; (Continued below).

Resveratrol from glucose by introducing heterologous enzymes tyrosine ammonia lyase, 4-Coumaryl Co-A ligase and resveratrol synthase. Further increase in levels were obtained by over expressing feedback insensitive DAHP synthase and Chorismate mutase and improving the precursor malonyl Co-A supply by over expressing acetyl-CoA carboxylase; short chain branched fatty acids by optimizing the native pathway, deletion of key genes in competing pathways and over expression of ATP binding cassette transporter; lactic acid production by expressing heterologous lactate dehydrogenase simultaneously attenuating glycerol-3phoisphate dehydrogenase and Cytochrome -coxidoreductase 2. Further increase in lactic acid levels were achieved by disrupting pyruvate decarboxylase and alcohol dehydrogenase and expressing acetylating acetaldehyde dehydrogenase; 1- hexadecanol by a combination of approaches Viz; over expressing fatty acyl Co-A reductase from barn owl (Tyto alba), over expression of acetyl Co-A carboxylase, increasing acetyl Co-A supply by expressing a heterologous ATP-dependent citrate lyase and knocking out of the negative regulator of Inositol-3-phosphate synthase involved in phospholipid metabolism; 3-hydroxypropioninc acid from beta alanine by expressing Beta-alanine -pyruvate amino transferase form bacillus cereus; $\mathrm{Rh} 2 \& \mathrm{Rg} 3$ are ginsenosides having anticancer activity whose production requires glycosylation of their aglycone precursor protopanaxadiol. Glcyosylation has been achieved by cloning and expressing genes for two UDP-glycosyltransferases from Panax ginseng; lycopene by different approaches Viz; obtaining exclusively phytoene synthase activity by inactivating the lycopene cyclase activity of the bi-functional enzyme form Xanthophyllomyces dendrorhous and expressing in this strain geranylgeranyl diphosphate synthase, phytoene desaturase and 3-hydroxy-3methylglutaryl Co-A reductase; 2,3-butanediol by a combination of different approaches Viz; introduction of heterologous biosynthetic pathway, by over expressing acetolactate synthase, alpha-acetolactate decarboxylase and 2,3-butanediol dehydrogenase and by redox balancing by over expressing water forming NADH oxidase; Overexpressing $p$-coumaric acid by combining different approaches Viz; reduction of aldehyde by-products by knocking out phenylpyruvate decarboxylase and pyruvate decarboxylase, over expression of feedback resistant DAHP synthase (3-deoxy-D-arabinoheptulosonate-7-phosphate), Chorismate mutase, Tyrosine ammonia lyase and shikimate kinase II; 
Engineering of the following pathways /products: muconic acid production by expressing heterologous enzymes, dehydroshikimate dehydratase, protocatechuic acid decarboxylase and catechol 1,2 -dioxygenase coupled with over expression of transketolase and a feedback resistant phosphor-2-dehyro-3-dehydroxyheptonate aldolase and deletion of glucose -6-phospahte 1 dehydrogenase; fatty acid synthesis by inactivation of isocitrate dehydrogenase and over expression of ATP-Citrate lyase; production of Protopanaxadiol (aglycon of many dammarane -type Ginsenosides having anticancer activity) by cloning and expressing dammarendiol-II synthase and protopanaxadiol synthase of Panax ginseng and NADPH-cytochrome P-450 reductase of Arabidopsis thaliana. Further increase in the levels were achieved by over expressing truncated 3hydroxy-3 - methylglutaryl-CoA reductase farnesyl diphopshate synthase, squalene synthase and 2,3 oxidosqualene synthase and codon optimization of protopanaxadiol synthase.

As mentioned above under $E$. coli here a little different expression of the contents --Over expressing glycerol -3phopshate dehydrogenase increases glycerol production though not glycerol -3-phopshatase; Increasing intracellular 1-glycerol-3-phopshate by over expressing glycerol-3phopshate dehydrogenase and reducing Pyruvate decarboxylase activity; malolactic fermentation is required to deacidify wine. To facilitate this heterologous malate permease and malolactic enzyme was introduced thereby achieving full decarboxylation of malate; Over production of native acetaldehyde dehydrogenase and heterologous acetylCo-A synthetase increases mevalonate pathway thereby resulting in Increased amorphadiene (sesquiterpene precursor to antimalarial drug artemisinin); complete biosynthetic pathways for flavonoid products, resveratrol, naringenin, genistein and kaempferol and quercetin were constructed by expressing genes obtained from different plants; over expression of Xylitol dehydrogenase enzyme reduces Xylitol accumulation and increases ethanol accumulation; over expression of NAD dependent $\mathrm{d}$-Xylose dehydrogenase enzyme reduces Xylitol accumulation and increases Xylonate accumulation; Over expression of heterologous genes Xylose isomerase, Xylose kinase and for non-oxidative pentose phosphate pathway increases ethanol production form Xylose; ATP independent acetyl Co-A synthesis was achieved by replacing acetyl Co-A synthase with bacterial acetylating acetaldehyde dehdyrogenase and pyruvate formate lyase;

\subsection{Corynebacterium Glutamicum}

Increasing the levels/production of the following: glutamate by increasing culture temperature from 33.0 to 39.5 Centigrade over a 5 min period. Changes in intracellular level of various metabolites occur which in turn can affect glutamate production with lactate decreasing the glutamate formation significantly; trehalose by expressing $E$. coli UDP -glucose pyrophopshorylase; lysine by over expression of fructose 1,6 bisphosphatase when grown on sucrose but not with glucose and/or fructose; methionine by replacing sulfate by thiosulfate or sulfide which was further increased by supplementing with methanethiol; methane levels by expressing threonine feedback insensitive homoserine dehydrogenase and deletion of homoserine kinase; Leucine by a combination of approaches Viz; expressing feedback resistant isopropylmalate synthase and acetohydroxysynthase, over expression of leuBCD (3isopropylmlate dehydrogenase and isopropylmalate isomerase), increasing glucose uptake; lysine by replacing NAD-dependent glyceraldehydes -3-phosphate dehydrogenase with NADP-dependent glyceraldehydes -3phosphate dehydrogenase - that is by altering the specificity of the enzyme form NAD towards NADP by enzyme (protein) sequence manipulation; valine production by deletion of pyruvate dehydrogenase complex, alanine amino transferase and threonine dehydrates and over expression of acetohydroxy synthase, acetohydroxyacid isomero reductase, leucine responsive protein and genes responsible for the export of three branched chain amino acids and methionine; Itaconate by expressing heterologous cis-aconitate decarboxylase. Further increase in production was achieved by fusing the enzyme with maltose-binding protein, reducing the isocitrate dehydrogenase activity and limiting nitrogen supply; Ethylene glycol by two different pathways from serine by expressing amino acid dehydrogenase/amino transferase and alpha keto acid decarboxylase and also by expressing ethanolamine decarboxylase, monoamino oxidase and alcohol dehydrogenase. Further enhancement was achieved by engineering serine biosynthetic pathway; lysine production by replacing endogenous gyceraldehyde-3phosphate dehydrogenase (gapA) with a heterologous nonphosphorylating NADP dependent glyceraldehydes 3 phosphate dehydrogenase (gapN) and subsequently express gapA under the control of myoinositol inducible promoter and expressing desensitized aspartokinase; (Continued below).

Arginine by inactivating two NAD (P) H-dependent hydrogen peroxide -forming flavin reductases deletion of NADH oxidase and AMP nucleosidase and increasing ATP \& NADH levels by over expression of 3-phosphoglycerate kinase and pyruvate kinase; methanol utilization by adaptive laboratory evolution and enhancing glutamate production; Six genes involved in oxaloacetate and $\alpha$-ketoglutarate utilization were manipulated and dynamically modulated the activity of the $\alpha$-ketoglutarate dehydrogenase complex to increased the production of 4-hydroxyisoleucine; glutaric acid by utilizing both native and heterologous genes coding for 4-aminobutyrate amino transferase, succinate semialdehyde dehydrogenase, 5-aminovalerate transaminase, glutarate semialdehyde dehydrogenase, lysine 2monooxygenase and delta-aminovaleramidase; Hyaluronic acid biosynthesis by attenuation of the glycolysis, pentose phosphate pathways and the dehydrogenation of pyruvate and knock out of lactate/acetate pathway; gammapolyglutamic aicd by introducing genes encoding $\gamma$-PGA synthase complex from $B$. subtilis (pgsB, C, and A) or $B$. 
licheniformis (capB, C, and A) were expressed under inducible tac in a native strain of L-glutamic acid producer. Strain F343 carrying capBCA produced higher $\gamma$-PGA compared with F343 expressing pgsBCA;

As mentioned above under $E$. coli here a little different expression of the contents: altering levels of NADPH produced through pentose phosphate pathway and TCA cycle by replacing native NADPH-dependent glutamate dehydrogenase with heterologous NADH-dependent Glutamate dehydrogenase; Temperature triggering of glutamate production was also influence by activities of phopshoenolpyruvate carboxylase, pyruvate carboxylase and biotin concentrations. Over expression of PEP carboxylase under biotin limiting conditions increases glutamic acid levels; expression of feedback resistant aspartate kinase coupled with leucine limitation enhanced lysine production. NADPH regeneration through pentose phosphate pathway is essential for increased lysine production; simultaneous amplification of pyruvate carboxylase and aspartate kinase increases lysine production to higher levels than expression of either gene alone; Glutamate production by $C$. glutamicum was higher than that by $C$. efficiens. In both strains, the decrease in the ODHC (2-oxoglutarate dehydrogenase complex) specific activity is responsible for increased glutamate production; deletion of pyruvate kinase enhances both glutamate and aspartate production; Over expression of feedback resistant threonine dehydratase or acetohydroxy synthase enhances isoleucine production which is further enhanced if both enzymes are co-expressed; Increase production of lysine by deleting succinate Co-A ligase; Triacylglycerol biosynthesis was achieved by engineering a complete pathway by the cloning and expression of 14 heterologous genes and elimination of by-products acetic acid and lactic acid by two more genes; production of Shikimic acid by a combination of approaches Viz; inactivation of shikimate kinase and other reactions involving the consumption of shikimate and its precursor dehydroshikimate, increasing the precursor PEP supply by over expressing myo-inositol transporter and glucokinase and inactivating PEP: sugar phosphotransferase, eliminating the unwanted products by expressing glyceraldehydes 3phospahte dehydrogenase and blocking dihydroxyacetone synthesis;

\subsection{Other Microbial Hosts and Products}

As mentioned above major bulk of the work involves $E$. coli followed by Sachhromyces cerevisiae and Corynebacteirum glutamicum. Of course many more microorganisms -bacteria, fungi, algae, cyanobacteria and diatoms - have been studied in relation to metabolic engineering but their individual coverage as a proportion of the total is less and their many products are identical/similar in category to the above described three microorganisms. Hence, not to make the manuscript too exhaustive and unwieldy for readers and to reduce overlapping and redundancy only a mention of different microorganisms and products studied is made in an alphabetical order as below:

\section{Host Microorganisms}

Acetobcterium woodii; Aeromicrobium erythreum; Ashbya gossypii; Aspergillus nidulans; Aspergillus niger; Azotobacter vinelandii; Bacillus subtilis; Candida famata; Candida glabrata;

Chlamydomonas; Clostridium acetobutylicum; Clostridium autoethanogenum; Clostridium beijerinckii; Clostridium pasteurinum; Clostridium thermocellum; Clostrifium tributyricum;

Corynebacterium lactofermentum; Cupriavidus necator; Geobacillus thermoglucosidasius;

Halomonas bluephagenesis; Klebsiella pneumonia; Klebsiella oxytoca; Kluveromyces lactis;

Lactobacillus lactis; Mannheimia succiniproducens; Neurospora crassa; Penicillium chrysogenum; Penicllium patulum; Phaeodactylum tricornutum; Pichi pastoris; Propionibacterium freudenreichii; Pseudomonas aeruginosa; Pseudomoans putida; Ralstonia eutrophia; Rhizopus oryzae; Rhodococcus sps; Sachropolysposra erythrea; Sphingomonas sps;

Streptomyces ansochromogenes; Streptomyces cinnamonensis; Streptomuces clavuligerus; Streptomyces coelicolor; Streptomyces lividans; Streptomuces tsukuniensis; Streptomyces venezeluae; Synecochoccus sps; Synechocystis sps; Synechocystis elongates; Ustilago maydis; Canthomonas campestris; Yarrowia lipolytica; Zymomonas mobilis;

\subsection{Products and Processes Studied}

1,2-propanediol; 1,3, propanediol; 1-butanol; 1-decanol; 2,3-Butane diol; 2-ketoisovalerate; 2-methylcitric acid; 3hydroxy propionate; Alginates with different sequence distribution of Beta-D -Mannuronic acid and alpha-Lguluronic acid; Astaxanthin; Branched chain higher alcohols; Butyric acid; Carotenoid accumulation; Cephalosporin; Citrate; Clavulanic acid; Cyanophycin; Daunorubicin; Docosohexanoic acid; Ectoine; Eryhtromycin; Ethanol; Fatty acids; FMN; Folate; Fumaric acid; Glutathione; Hyaluronic acid; Isobutanol; Isoprenes; Isopropanol; taconate; Lactic acid; Lysine; Malate; Mannitol; Methylketone; Monensin A \& B (polyketides); N-acetylglucoseamine; Penicillin; PHA (polyhydroxy alkanoates); Polyhydroxy butyrate; Propionic acid; PUFA; Resveratrol; Riboflavin; Threonine; Triglycerides; Vitamin B11; Vitamin B2; Xanthan; $\beta$ - lactam; As in the above three microorganisms products include Antibiotics-amino acids, organic acids, fatty alcohols, branched chain alcohols, sugars \& sugar alcohols, pigments, different secondary metabolites etc apart from manipulating / studying metabolic fluxes in glycolysis, glyoxylate cycle, central metabolism and secondary metabolism, nutrient and oxygen utilization.

\section{Metabolic Engineering of Plants}

Plants are of agricultural, ecological and pharmaceutical significance. Importance of Plants cannot be overemphasized 
and hence of Plant Metabolic Engineering. However, Plant metabolic engineering is far more complex and difficult compared to simple microbes. Metabolic engineering studies have been carried out to increase seed biomass and biomass of other parts to provide for more food, feed, fiber and fuel both under normal and varied environmental conditions.. Further, plants are rich in secondary metabolism and secondary metabolites play an important role in providing many bioactive compounds for health care, plant defense against biotic stresses and environmental cleaning, different colors, aromas and flavors. In view of the above in addition to fulfilling the basic needs of humans and animals they serve pharma and chemical industries $[15,16]$.

In the above context, metabolic engineering studies with the above mentioned tools and techniques have been carried out on the following specific aspects: a) Manipulating primary (central) metabolism and secondary metabolism. In primary metabolism focus has been on: increasing carbohydrate storage capacity, increase fatty acid biosynthesis, increasing EFA (essential fatty acids) omega-3 fatty acids \& long chain PUFA (Polyunsaturated fatty acids) Eicosapentaenoic acid and docosahexaenoic acid; increasing triglycerides (oil) and wax; increasing the level of essential amino acids (lysine, methionine, tryptophan) and protein content and increasing the biological value of seed storage proteins; increasing ht level of non-standard amino acid (LDOPA (L-3,4 Dihydroxy phenylalanine) a drug for parkinsons; increase the levels of osmoprotectants (metabolites which protect plants from drought and salinity stresses, Ectoine, Glycine betaine, Trehalose. Polyols); study of carbon partitioning into sucrose, starch and cellulose; manipulation of membrane transporters. As pant cells are highly compartmentalized and different pathways and different reactions take place in different compartments passing in and out of metabolites from different compartments is a normal phenomenon in plants. Hence, manipulating membrane transporters is an important strategy; study of micronutrient metabolism; potential for phytoremediation.

In secondary metabolism the focus has been on / to understand the relative contribution of MVA (mevalonic acid) and MEP (Methyl erythritol 4-phosphate) pathways towards synthesis of isoprenoids; increasing different flavonoids (Baicalin, Baicalein, Wogonin, Genestein,) and anthocyanins, increasing different alkaloids (Vincristine, Vinblastine, Serpentine, Tryptamine), increasing different carotenoids (beta -carotene, lycopene, crocin, picrocrocin, ketocarotenoids,) and xanthophylls (astaxanthin, zeaxanthin, lutein); increasing terpenoids (nerol, neral, geranial, geranic acid, tanshinone, paclitaxel (taxol, notoginsonide R1); increasing phenylpropanoids (eugenol, isoeugenol); increase in tocopherols (with vitamin E activity) \& tocotrienols (deltatocotrienol). Together they are referred to as tocochromanols; increasing the degradation of toxic xenobiotics PAH (Polycyclic aromatic hydrocarbons); increasing 1,2,4Butanetriol an important building block for different drugs and chemicals.
In the above endeavors apart from model crops Tobacco (Nicotiana benthamiana \& N. tabacum)), Maize (Zea mays), Arabidopsis (Arabidopsis thaliana), Rice (Oryza sativa), Soybean (Glycine max) Tomato (Solanum lycopersicum), Birdsfoot trefoil (Lotus japonicus), Lettuce (Lactuca sativa) Banana (Musa sps), Potato (Solanum tuberosum, Solanum phureja) Petunia (Petunia hybrida) Strawberry (Fragaria vesca), Panax (Panax notoginseng) have been explored for metabolic engineering. In addition to intact genetically engineered plants of the above hairy root cultures of Scutellaria (Scutellaria baicalensis), Catharanthus (Catharanthus roseus) \& Red sage /Danshen (Salvia miltiorrhija) and Taxus (Taxus cuspidata) suspension cell cultures have been studied.

A point to be noted is, genes used in metabolic engineering are not always from the same plant but can be from other plants and different microorganisms. Genes coding for different enzymes and other functional proteins are selected rationally based on the pre-existing knowledge of metabolic pathways in and regulatory/rate limiting steps in different organisms. Their list is as follows:

UFP-glycosyltransferase, UDP-glucose dehydrogenase, UDP-rhamnose synthase 2,

UDP-D-Xylose epimerase. PAP1 Transcription factor, Tryptophan decarboxylase, Anthranilate synthase-alphasubunit, Geranylgeranyl diphosphate dehydrogenase, Homogentisate dioxygenase from Arabidopsis thaliana; Phosphomevalonate kinase, Mevalonate diphosphate decarboxylase, Hydroxymethylglutaryl CoA Synthase Hydroxymethylglutaryl CoA reductase, starch/glycogen synthase from Sacchromyces cervisiae; Hydroxymethylglutaryl CoA reductase, 1-deoxy-Dxylulose-5 - phosphate synthase, Geranylgeranyl diphosphate synthase from Salvia miltiorrhiza; Betaine aldehyde dehydrogenase, Xylonate dehydratase from $E$. coli; Neryldiphosphate synthase 1, Lycopene -epsiloncyclase from tomato and Lycopene -epsilon-cyclase from banana; Benzoylformate decarboxylase, Benzoylformate dehydrogenase from Pseudomonas putida; Starch Branching Enzyme, Starch /Glycogen synthase from Cynidio merolae; Beta-carotene ketolase, Beta-carotene hydroxylase from Haematococcus pluvialis; Polyphenol Prenyltransferases from Streptomyces Sps/S. coelicolor/ Sophora flavescens; Eugenol synthase \& Isoeugneol synthase from Wild strawberry (Fragaria vesca); Isoflavone synthase, Phenylalanine ammonia lyase from soybean; Choline monooxygenase, Choline oxidase, Anthocyanidin synthase, ORCA 3 (octodecanoid responsive catharnahtus AP2 domains) transcription regulator, Polyphenol prenyltransferases, Prephenate dehydrogenase, Naphthalene dioxygenase system, Tyrosine hydroxylase, Carotenoid cleavage dioxygenase, Phytoene synthase, Xylose dehydrogenease, Beta-carotene hydroxylase, Geraniol synthase, from Arthrobacter pascens, Rice, Catharanthus roseus, Erwinia herbicola, Mycobacterium vanbaalenii, Croccus sativus \& Buddleja davidii, Pantoea ananatis, Caulobacter vibriodes, Saffron 
and Lippia dulcia respectively.

(Note of caution-The above list is only confined to the papers scanned in the issues of "Metabolic Engineering". These papers do quote many other plant systems in their review of literature which have been studied and published in several other journals. A good overview of tools, techniques, methodologies, products, genes sources from different organisms etc can be obtained from the special issue on Plant Metabolic Engineering).

\section{Metabolic Engineering of Mammalian/Animal Systems}

As mentioned above metabolic engineering in animal and human systems are less covered. Studies are mainly focused on cell culture systems. These cell culture systems are widely used for both basic research and therapeutic solutions and industrial applications. Animal cells are widely used to produce products relevant to animal and human health care. Important systems include: Hepatocytes of different organisms -rat, pig, human, particularly of rat, $\mathrm{CHO}$ (Chinese Hamster Ovary) cells, HEK (Human Embryonic Kidney)- 293, BHK (Baby Hamster Kidney) cells, HT-1080 (Human fibrosarcoma cell line), NSO murine cells (nonsecreting murine myeloma murine cells) in addition to liver and adipose tissues and intact animals [17-20].. Both parental cell lines and derived cell lines are used. These mammalian cells are commonly used for the commercial production of many therapeutic proteins and vaccines. Mammalian cells mentioned above are important manufacturing platforms in bioengineering. In this context, it may be mentioned that designing improved apoptosis inhibitors is of immense importance for biotechnological applications as apoptosis is a serious problem in the commercial production of products form cell culture. (Of course a point to be remembered is that studies on microbial and plant metabolic engineering are concerned with the production of pharmaceuticals/therapeutics for various ailments). Broadly the above mentioned systems were studied in relation to different aspects as follows:

\subsection{Rat Hepatocytes}

(APR (Acute Phase Response) and expression of APP (Acute Phase proteins) under increased temperatures and influence of cytokine IL-6 (Interleukin); Effect of Interleukin-1b and Interleukin-6 on perturbations in mitochondrial metabolism and hepatic dysfunction; reducing apoptosis by over expressing anti-apoptotic genes; Up regulation of glucose, fatty acid and amino acid turnover in isolated perfused livers of burned rats; Controlling thrombosis in rat artery by anti-thrombotic activity of catalase and chondroitin sulfate; Controlling apoptosis by over expression of anti-apoptotic genes with the aim to generate more efficient cells with higher productivities; Induction of hyper-metabolic state by the treatment of hydrogen peroxide and glucagon to understand the inflammatory responses; atorvastatin -a hyper-lipidemic drug - decreasing the flux through cholesterol pathway but not affecting the central metabolism significantly. Porcine and rat hepatocytes were studied with respect to oxygen consumption with the aim of designing bioartificial liver assist devices.

\subsection{Human Adipocytes}

Altered triglyceride metabolism by perturbing pyruvate metabolism using inhibitors of lactate dehydrogenase and pyruvate carboxylase in 3T3-L1 cells. Reduction in triglyceride accumulation attributed to reduction in the de novo fatty acid synthesis.

\subsection{HEK-293}

HEK-293 parental and derived cell lines are highly useful heterologous systems for the development of recombinant therapeutic proteins, vaccines and many drugs, production of viral vectors like adenovirus, adeno-associated virus, lentivirus etc and studies on structural biology and electrophysiology). Central metabolism related to glucose, and almost all amino acids -in particular glutamine- has been studied extensively in 293 parental cell lines under different culture conditions. Unlike the above, a derived cell line produces as a result of metabolism lactate and in a particular ammonia which are toxic to the growth of the cells; enhancing adenovirus reproduction in a derived cell line (293SF-3F6) by improving the physiological state of the cells, to meet the high demand of Adenovirus for gene therapy programs; Validating the concept of extending the in vivo circulation time of recombinant glycoproteins by improved sialylation using erythropoietin as a reference protein and $31 \mathrm{~N}$-glycosylation related genes in different mammalian cells in addition to HEK-293, Viz; (CHO, BHK, Cos-7 (Derived from African Green Monkey Kidney, acronym "COS" is derived from the cells being CV-1 (simian) in Origin, and carrying the SV40 genetic material.), 3T3 (Mouse embryonic fibroblasts. 3T3 is a designation that stands for passaging the cells 3 times/week at 1:3), NSO cells); reduction of toxic metabolites lactate and ammonia by over expressing Pyruvate carboxylase gene leading to increased production of recombinant IFN-alpha2b (Interferon);

\subsection{CHO Cells}

Down regulation and activity of branched chain amino acids catabolism by over expression of a branched-chain $\alpha$ ketoacid dehydrogenase (BCKD) to control muscle protein degradation under different disease conditions; Inhibiting apoptosis by over expression of the genes Bcl-2 (B Cell lymphoma 2) and its homologues Bcl-xL and its mutant Bcl$\mathrm{xL}$ devoid of a long segment of 58 amino acids; toxicity of ammonium altering the glycosylation profile of recombinant proteins by decreasing the activity of glycosyl transferases; Regulation of apoptosis by many proteins in different compartments of the cell Viz; cytosol, mitochondria and 
endoplasmic reticulum and reducing apoptosis by using different media; induction of Heparin (most commonly used blood coagulant) pathway by transfecting human Ndeacetylase/N-sulfotransferase (NDST2) and mouse heparan sulfate 3-O-sulfotransferase 1 (Hs3st1) genes into $\mathrm{CHO}$ host cells growing in suspension culture and establishing stable CHO cell lines expressing both NDST2 and Hs3st1. Need for further research emphasized as Heparin sulfate is not as much comparable to Heparin; by extensive mitochondrial DNS sequencing it was established heteroplasmy (variation in organelle genome) is widespread in CHO cell lines and that better biopharmaceutical manufacturing is possible through rational mitochondrial genetic engineering; cells were engineered to reduce the formation of toxic metabolites, lactate and ammonia by over expressing four genes in the biosynthesis of Phenylalanine \& tyrosine and knocking out catabolic enzyme branched chain amino transferase 1 (BCAT 1) which has resulted in improved cell growth \& productivity and overall culture process performance; An effort has been made to develop new $\mathrm{CHO}$ cell expression systems capable of producing higher level of secretory recombinant biotherapeutics along with improved product quality. In this endeavor global transcriptional activator of genes involved in lipid biosynthesis, sterol regulatory element binding factor 1 (SREBF1), and stearoyl CoA desaturase 1 (SCD1), which catalyzes the conversion of saturated fatty acids into monounsaturated fatty acids were overexpressed in $\mathrm{CHO}$ cells to different degrees which has led to enhancement of secretory recombinant protein products;

Knockout of sialidase three sialidase genes (Neu 1, 2, and 3 ) and two pro-apoptotic genes $\mathrm{BAK}(\mathrm{Bcl}-2$ homologous antagonist killer) and BAX (Bcl-2-associated $\mathrm{X}$ protein) in Chinese hamster ovary cells enables the production of heavily sialylated recombinant proteins as demonstrated for recombinant tetra-sialylated human erythropoietin (rhEPO);

\subsection{Liver \& Adipose Tissue}

Differential contributions of exogenous glucose and fructose towards synthesis of saturated and unsaturated fatty acid (oleate) and glycerol-3- phosphate was assessed. Fructose was found to be more lipogenic particularly in liver.

Gene therapy is holding lot of potential and promise to cure diseases. The process involves studying critically the metabolism of the diseased tissue/ organ and identify genes which can be introduced to cure the disease. Genes are delivered into the host tissues by different viral vectors like adenoviral, retroviral etc and also by non- viral vectors/methods like liposomes, DNA-Protein conjugates etc. to restore normal condition. Gene therapy with good success carried out in Cystic fibrosis, Cancer and Diabetes [18].

\section{Conclusions}

A glimpse of metabolic engineering in microbial, plant \& mammalian/animal systems has been presented as outlined section 1. A primary aim is to motivate policy makers to start relevant International Research Institutes on (Metabolic
Engineering). One way to accomplish this is on the pattern of CGIAR (Consortium (formerly consultative group) of International Agricultural Research Institutes which has 15 institutes across the world. CGIAR headquarters is in Montpellier, France. (Readers should be aware that presently we do have ICGEB (International Centre for Genetic Engineering and Biotechnology) with head Quarters at Trieste, Italy and a branch in New Delhi, India. However, as it clearly evident from the manuscript and the papers published in the exclusive journal, "Metabolic Engineering" is much more a gigantic task and the returns also can be enormous.. Hence, the need to establish specific International Institutes focusing on metabolic engineering and to fulfill the ambition of "Next Industrial Biotechnology Revolution". Manuscript gives a glimpse of various categories of products and the processes and tools \& techniques etc employed for metabolic engineering in different living systems.

\section{Conflict of Interest}

Author has no conflict of interest.

\section{Acknowledgements}

My sincere thanks especially to Dr S. Sathish, who helped me in literature survey. I also thank Dr G. Satish and Dr Anil kumar Moola for helping me in the preparation of the manuscript. Above are from Departments of Biotechnology and Plant science.

\section{Note}

(This paper has been written after scanning through the issues from the journal inception -1999 January (Volume 1) till January 2021 (Volume 63)-21 years. In all they constitute 63 volumes, 122 issues and 15,700 plus pages. Total number of articles (papers) in the above are approximately 1600. It is evident that the average length of every paper is fairly long. It is not possible to give a s many references. Selected references have been given. Readers are advised first to go through "Special Issues" (Ref. Nos; 4-8 \& 15-17 followed by "Reviews", Ref. No. 9-11, followed by some selected articles Ref. No. 12-14 \& 18-20).

\section{References}

[1] https://www.journals.elsevier.com/metabolic-engineering

[2] Bailey, J. E. (1991). Toward a science of metabolic engineering. Science, 252 (5013), 1668-1675. doi: $10.1126 /$ science. 2047876 .

[3] https://en.wikipedia.org/wiki/Metabolic_engineering

[4] Hal Alper (Ed) (2021) Tools and Strategies of Metabolic Engineering Volume 63, 1-160. 9 articles.

[5] Hal Alper (Ed) (2018) Metabolic Engineering Host Organism. 50, 1-208. 12 articles. 
[6] Ka-Yiu San (Ed) (2008) Engineering Metabolic Pathways for Biofuels Production 10, 293-404. 11 articles.

[7] Hal Alper (Ed) (2020). Metabolic Engineering Products. 58, $1-132.6$ articles.

[8] Blaine Pfeifer and Robert Kiss (Ed) (2010) Metabolic Flux Analysis for Pharmaceutical Production. 12, 81-172 9 articles.

[9] Bongaerts, J., Krämer, M., Müller, U., Raeven, L. and Wubbolts, M. (2001). Metabolic Engineering for Microbial Production of Aromatic Amino Acids and Derived Compounds. Metabolic Engineering, 3, 289-300. doi: $10.1006 /$ mben.2001.0196

[10] Thykaer, J. and Nielsen, J. (2003). Metabolic engineering of $\beta$-lactam production. Metabolic Engineering, 5 (1), 56-69. doi: 10.1016/s1096-7176(03)00003-x.

[11] Handke, P., Lynch, S. A. and Gill, R. T. (2011). Application and engineering of fatty acid biosynthesis in Escherichia coli for advanced fuels and chemicals. Metabolic Engineering, 13 (1), 28-37. doi: 10.1016/j.ymben.2010.10.007.

[12] Trantas, E., Panopoulos, N. and Ververidis, F. (2009). Metabolic engineering of the complete pathway leading to heterologous biosynthesis of various flavonoids and stilbenoids in Saccharomyces cerevisiae. Metabolic $\begin{array}{llll}\text { Engineering, } & 11 & (6), & 355-366\end{array}$ 10.1016/j.ymben.2009.07.004.

[13] Zhang, X., Tervo, C. J. and Reed, J. L. (2016). Metabolic assessment of E. coli as a Biofactory for commercial products. Metabolic Engineering, 35, 64-74. doi: 10.1016/j.ymben.2016.01.007.
[14] San, K.-Y., Bennett, G. N., Berríos-Rivera, S. J., Vadali, R. V., Yang, Y.-T., Horton, E., and Blackwood, K. (2002). Metabolic Engineering through Cofactor Manipulation and Its Effects on Metabolic Flux Redistribution in Escherichia coli. Metabolic Engineering, 4 (2), 182-192. doi: 10.1006/mben.2001.0220.

[15] Plant Metabolic Engineering --Metabolic Engineering (2002) 4, 1-106.12 articles (Editor not mentioned).

[16] Sandmann, G., Römer, S. and Fraser, P. D. (2006). Understanding carotenoid metabolism a necessity for genetic engineering of crop plants. Metabolic Engineering, 8 (4), 291302. doi: 10.1016/j.ymben.2006.01.005.

[17] Christian Metallo and Ralph Deberardinis (Ed) (2017). Engineering approaches to study cancer metabolism. 43: Part B, (Special Issue) 93-226), 12 articles.

[18] Wang, F., Raab, R. M., Washabaugh, M. W. and Buckland, B. C. (2000). Gene Therapy and Metabolic Engineering. Metabolic Engineering. 2 (2), 126-139 doi: 10.1006/mben.1999.0144 139 .

[19] Joaquim Vives, Sandra Juanola, Jordi Joan Cairó, Francesc Gòdia, (2003). Metabolic engineering of apoptosis in cultured animal cells: implications for the biotechnology industry. 5 (2), 124-132. doi: 10.1016/s1096-7176(03)00024-7.

[20] Zhang, P., Lifen Tan, D., Heng, D., Wang, T., Mariati, Yang, Y., \& Song, Z. (2010). A functional analysis of Nglycosylation-related genes on sialylation of recombinant erythropoietin in six commonly used mammalian cell lines. Metabolic Engineering, 12 (6), 526-536. doi: 10.1016/j.ymben.2010.08.004. 